\title{
Étude du genre Campanile Fischer, 1884 (Mollusca: Gastropoda : Campanilidae). 1. Description d'une espèce nouvelle du Miocène supérieur du Cap Amparafaka (Madagascar)
}

\author{
Jean-Michel Pacaud* \\ CR2P Centre de Recherche en Paléontologie-Paris, Muséum national d'Histoire naturelle, Sorbonne Université, CNRS, 8 rue Buffon, \\ CP 38, 75005 Paris, France
}

Reçu le 14 mai 2019 / Accepté le 29 mai 2020 / Publié en ligne: 22 décembre 2020

\begin{abstract}
Résumé - L'espèce Campanile madagasikara nov. sp. est décrite du Miocène supérieur du Cap Amparafaka à Madagascar. Le genre Campanile est signalé pour la première fois au Cénozoïque à Madagascar. En effet, le genre n'a pas jusqu'ici été rencontré à Madagascar, à part dans les sédiments crétacés. Il s'agit donc de l'occurrence la plus récente du genre jusqu'ici enregistré à Madagascar. Les Campanile au Miocène sont seulement présents en Asie du Sud-Est, en Nouvelle-Zélande, en Australie et en Afrique de l'Est. La découverte de l'espèce malgache décrite ici suggère l'existence d'aires relictes dans l'océan Indien et le Pacifique au Néogène et jusque dans l'actuel en Australie. L'identité des cordons spiraux est recherchée par leur correspondance topologique et ontogénétique, puis codifiée par une terminologie. Cette méthode descriptive utilisée par Pacaud J-M, Ledon D, Loubry P et Fernandez S. (2014. Importance de la correspondance ontogénétique et topologique de la sculpture spirale dans la discrimination des espèces du genre Campanile (Mollusca, Gastropoda). Fossiles, Revue française de paléontologie 19 : 23-49, fig. 1-17, pl. 1-12) permet de clarifier la présentation des caractères et de proposer des homologies structurales.
\end{abstract}

Mots clés : Campanilidae / Campanile / Madagascar / Miocène supérieur / sculpture spirale / terminologie / taxonomie

Abstract - Study of the genus Campanile Fischer, 1884 (Mollusca: Gastropoda: Campanilidae). 1. Description of a new species from the Upper Miocene of Cap Amparafaka (Madagascar). The species Campanile madagasikara sp. nov. is described from the Upper Miocene of Cap Amparafaka, Madagascar. The genus Campanile is recorded for the first time to Cenozoic in Madagascar. Indeed, the genus has not hitherto been recorded from Madagascar, a part of Cretacous sediments. This is the most recent occurrence of the genus so far recorded from Madagascar. Miocene Campaniles are only found in Southeast Asia, New Zealand, Australia and East Africa. The discovery of the Malagasy species described here suggests the existence of relict areas in the Indian Ocean and the Pacific at the Neogene and even at present in Australia. The identity of the spiral cords is researched by their topological and ontogenetical correspondences, then codified by a terminology. This descriptive method used by Pacaud J-M, Ledon D, Loubry P and Fernandez S. (2014. Importance de la correspondance ontogénétique et topologique de la sculpture spirale dans la discrimination des espèces du genre Campanile (Mollusca, Gastropoda). Fossiles, Revue française de paléontologie 19: 23-49, fig. 1-17, pl. 1-12) allows to clarify the presentation of the characters and to propose structural homologies.

Keywords: Campanilidae / Campanile / Madagascar / Upper Miocene / spiral sculpture / terminology / taxonomy

\section{Introduction}

Depuis la reconnaissance approfondie de l'île de Madagascar par l'explorateur-naturaliste français Alfred Grandidier (1836-1921), l'île est bien connue pour ses dépôts fossilifères et héberge de nombreux sites du Crétacé supérieur parmi les

\footnotetext{
* Auteur de correspondance : pacaud@mnhn.fr
}

plus importants du monde. Des fossiles marins du Cénozoïque sont aussi décrits de Madagascar, mais ceux-ci ont reçu beaucoup moins d'attention de la communauté scientifique, la plupart de ces travaux datant du XIX ${ }^{\mathrm{e}}$ et des premières années du $\mathrm{XX}^{\mathrm{e}}$ siècle. Les dépôts marins cénozoïques de Madagascar ont été recensés, mais peu ont été systématiquement explorés. Certaines études publiées incluent quelques rapports sur l'Éocène et le Miocène et listent quelques invertébrés 


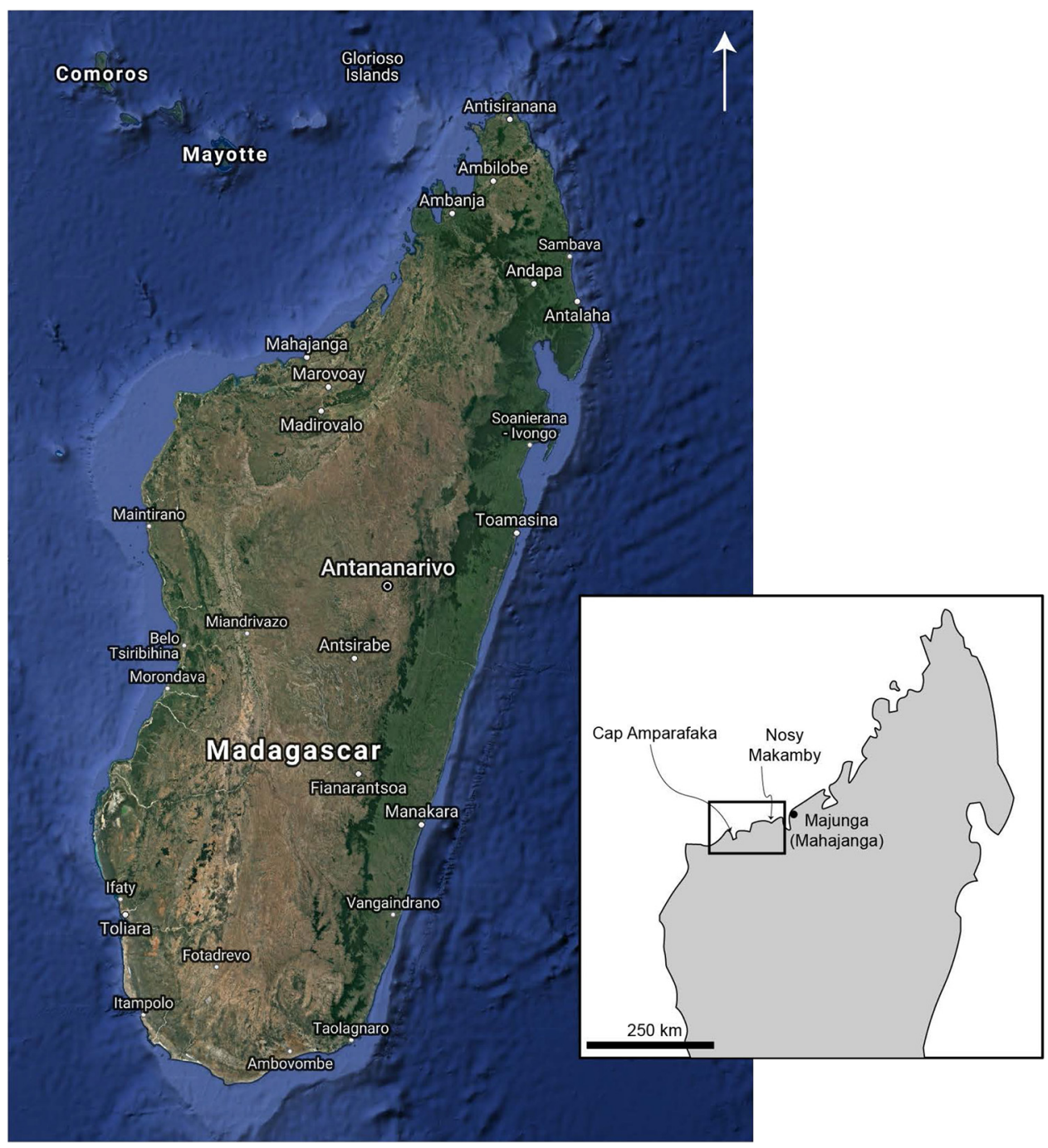

Fig. 1. Situation géographique (Map data (C) 2019 Google, Imagery (C) 2019 TerraMetrics).

Fig. 1. Geographic location (Map data () 2019 Google, Imagery (c) 2019 TerraMetrics).

(foraminifères, bivalves, gastéropodes, échinides) et des vertébrés (Collignon et Cottreau, 1927; Samonds et al., 2009; Andrianavalona et al., 2012). Historiquement, les dépôts miocènes ont été d'abord décrits de la partie occidentale et du Nord-Ouest de Madagascar (Perrier de la Bathie, 1921; Collignon et Cottreau, 1927; Hourq, 1949; Besairie, 1956; Besairie et Collignon, 1971). Perrier de la Bathie (1921) a publié le premier travail détaillé décrivant l'âge de ces formations post-crétacées et a donné les coupes géologiques de Nosy Makamby et du Cap Tanjona, deux des localités les mieux connues du Cénozoïque. Plus à l'ouest, Baron et Mouneyres (1904) ont publié une description géologique complète d'Amparafaka. Cependant, cette région reste mal décrite. Quelques auteurs ont noté la «présence de dépôts miocènes ", mais sans vraiment donner d'informations précises sur les localités (Collignon et Cottreau, 1927; Besairie et Collignon, 1971). Le Cénozoïque est donc relativement pauvre en fossiles d'invertébrés recensés : «les recherches pour cette période sont encore peu nombreuses et ponctuelles, concentrées sur quelques sites seulement (île Makamby, Besalampy, pointe Sada, cap Tanjona, Ampazony dans le Nord-Ouest). Elles ont été conduites là où des dépôts cénozoïques avaient été repérés par les auteurs historiques (Besairie, Collignon) : c'est dire que de nouvelles campagnes de prospection sont à entreprendre » (Rakotovao Andrianavah, 2015: 172).

Une espèce nouvelle de Campanile, récoltée par Raymond Decary, administrateur des colonies en 1922 et directeur de la recherche scientifique à Madagascar en 1937, est décrite ici du Miocène supérieur de la région côtière d'Amparafaka (Fig. 1). Par ailleurs, le genre Campanile est signalé pour la première fois au Cénozoïque à Madagascar. Ce genre est actuellement représenté par une seule espèce connue, Campanile symbolicum Iredale, 1917, vivant dans les eaux peu profondes des côtes du sud-ouest de l'Australie, sur des fonds sablonneux couverts d'algues (Ludbrook, 1971; Houbrick, 1981 ; Healey et Wells, 1998; Pacaud, 2012 : 79, pl. 4, fig. B-C). L'espèce vit en population importante par 1 à $4 \mathrm{~m}$ de fond et peut être partiellement ensablée près des rochers (Houbrick, 1984). Le genre Campanile n'a jusqu'ici été rencontré à Madagascar que dans les sédiments crétacés, avec des espèces telles que Campanile inauguratum 


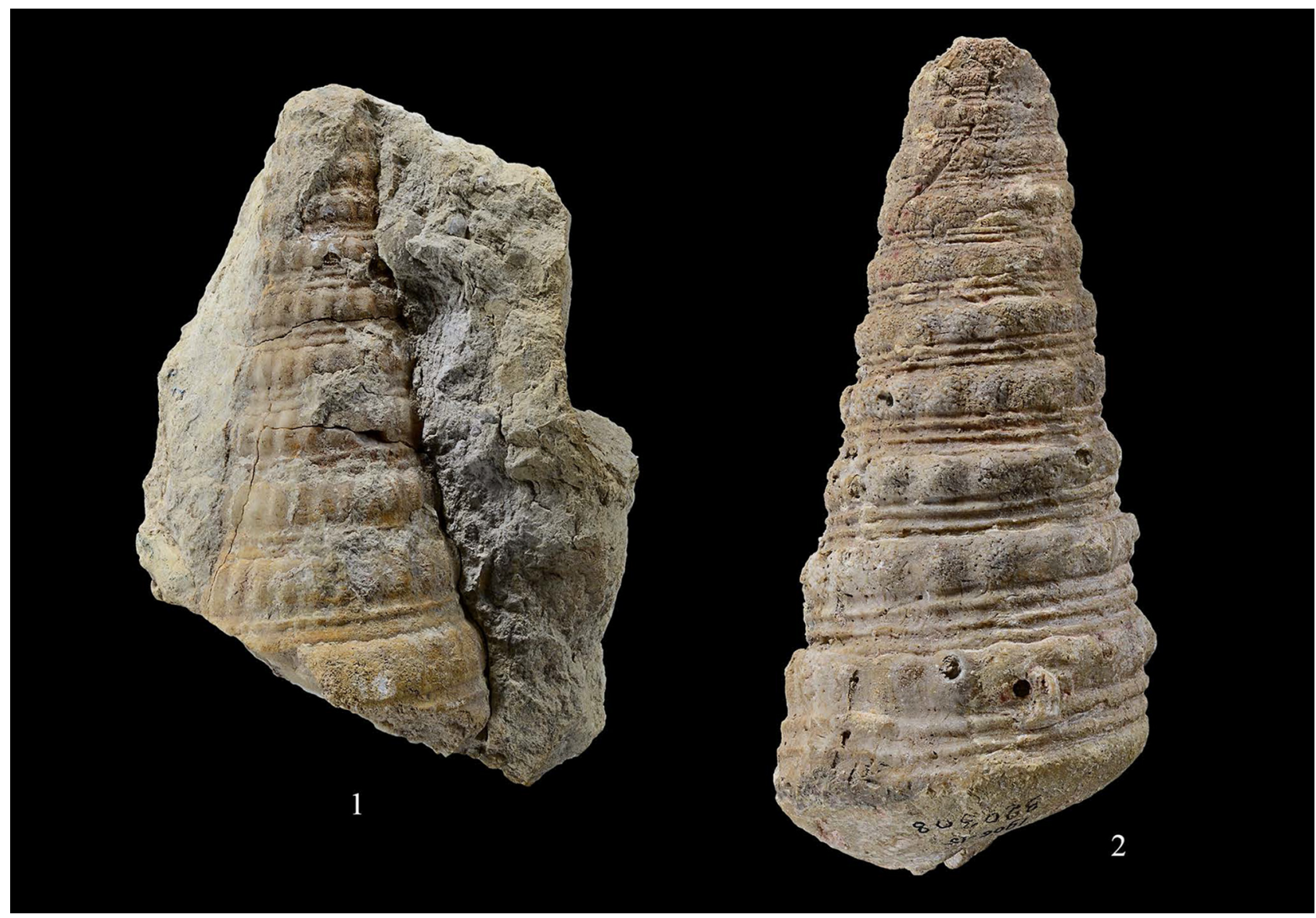

Fig. 2. Campanile inauguratum (Stoliczka, 1867). 1. Maastrichtien (Crétacé supérieur) de Belamoty (Madagascar), MNHN.F.B20308 (coll. Geay). Hauteur: 129,0 mm. 2. Santonien (Crétacé supérieur) des Tamarins (Madagascar). MNHN.F.A52130. Hauteur: 86,5 mm.

Fig. 2. Campanile inauguratum (Stoliczka, 1867). 1. Maastrichtian (Upper Cretaceous) of Belamoty (Madagascar), MNHN.F.B20308 (Geay coll.). Height: $129.0 \mathrm{~mm}$. 2. Santonian (Upper Cretaceous) of Tamarins (Madagascar). MNHN.F.A52130. Height: $86.5 \mathrm{~mm}$.

(Stoliczka, 1867) du Maastrichtien [MNHN.F.B20308 et A52130] (Fig. 2) (Douvillé, 1904 ; Cottreau, 1922; Basse, 1933 ; Delpey, 1942 ; Kiel et al., 2000).

Avec la présente note, nous débutons une série d'études taxonomiques sur le genre Campanile qui sera réalisée en suivant la méthode descriptive que nous avons établie (Pacaud et al., 2014). Nous étudierons soit des espèces nouvelles, soit des espèces incomprises ou mal interprétées.

\section{Contexte géographique et stratigraphique}

La région d'étude est localisée dans le bassin de Mahajanga, au Nord-Ouest de Madagascar. Cette région contient la coupe sédimentaire marine de dépôts du Miocène la plus complète de Madagascar, avec des extensions latérales qui affleurent dans les régions de Nosy Makamby, Cap Tanjona, Cap Sada et Amparafaka (Collignon et Cottreau, 1927). Amparafaka, dans la baie de Baly, est le site situé le plus au sud. Il est caractérisé par des falaises calcaires raides couvertes par des grès continentaux rouges du Pliocène. Fondé sur l'étude des mollusques fossiles, Collignon et Cottreau (1927) ont interprété l'âge des couches marines littorales de Nosy Makamby comme du Miocène inférieur (plus récent que l'Aquitanien), allant du Burdigalien à
l'Helvétien (l'ancien terme Helvétien équivaut approximativement à l'intervalle Langhien-Serravallien). Les Foraminifères Miliolidae sont abondants dans presque tous les dépôts de Makamby et suggèrent aussi un paléoenvironnement côtier tropical miocène (Lavocat et al., 1960; Ramihangihajason et al., 2014). Une étude récente (Ramihangihajason et al., 2014) sur les foraminifères benthiques, les ostracodes, les macro-invertébrés (bivalves, gastéropodes et échinides) ainsi que des vertébrés de Nosy Makamby et d'Amparafaka indique un âge Miocène supérieur. Les travaux sur les isotopes du strontium suggèrent que certains des sédiments peuvent être datés du début du Tortonien (Samonds et Fordyce, 2019).

\section{Matériel et méthode}

Le Campanile du Miocène de Cap Amparafaka, présentait une surface encroutée et a subi un traitement à l'hydroxyde de potassium $(\mathrm{KOH})$ pour le dégager de la gangue, nous permettant ainsi d'observer, sur une grande partie et très nettement, la sculpture spirale des tours. La méthode analytique pour identifier les homologies structurales des Calliostomatidae (Ikebe, 1942; Marshall, 1995), des Architectonicidae et des Mathildidae (Bieler, 1988, 1992, 1995), 
des Muricidae (Merle, 1999, 2001, 2005 ; Pacaud, 2018), des Turbinidae (Pacaud et Ledon, 2010), des Rostellariidae (Pacaud et al., 2011) et des Bursidae (Sanders et al., 2017, 2019) est ici utilisée. La méthode descriptive du type de sculpture spirale de cette espèce nouvelle est basée sur l'identification des cordons spiraux que nous avons mise en évidence (Pacaud et al., 2014) dans la morphologie des coquilles du genre Campanile. Cette méthode consiste à rechercher l'identité des cordons spiraux sur les espèces du genre Campanile par leur correspondance topologique et ontogénétique, puis à les codifier par une terminologie. L'étude de leur croissance permet ainsi de détecter les types de sculpture spirale de chaque espèce. Nous avions en effet noté que la méthode descriptive traditionnelle ne proposait aucune approche morphologique comparative pertinente, y compris sur l'identité des caractères de la sculpture spirale. Il est évident que le concept d'homologie pour ce type de caractères n'a jamais été appliqué pour discriminer les espèces du genre Campanile. La diversité de la sculpture spirale des jeunes Campanile est totalement méconnue, car, dans la majorité des cas, seuls les spécimens adultes sont décrits et illustrés. Ces derniers ne montrent en effet que rarement les cordons spiraux et ne présentent qu'une surface où ceux-ci ont fusionné avec le cordon spiral adapical (voir ci-après) et où seuls les tubercules ou les côtes sont observables. L'étude du développement sculptural des cordons spiraux n'avait jamais été appréhendée jusqu'à ce que nous ayons mis en évidence l'identification des cordons spiraux, à l'exception d'une ébauche d'analyse présentée par Wrigley (1940) qui avait bien saisi l'intérêt de l'étude de la position topologique des cordons spiraux. Les termes descriptifs utilisés jusqu'à présent dans la description des Campanile, à partir de la téléoconque, s'avéraient beaucoup trop imprécis; les cordons spiraux y étaient dénombrés mais jamais nettement identifiés. Sans une identification de la correspondance topologique des ornements (cordons, nodosité et épines) de ces espèces, il est impossible d'interpréter les descriptions classiques qui peuvent s'appliquer à de nombreuses espèces de Campanile.

Après avoir constaté chez les exemplaires juvéniles et subadultes des Campanile une variation importante dans le nombre de cordons spiraux et dans leur position, nous avons recherché les séquences d'apparition de ces derniers. Il y a seulement trois cordons primaires ([P1], [P2] et [P3]) sur les tours de spires subadultes de la coquille, auxquels d'autres cordons s'ajoutent par intercalation secondaire ([s1] et [s2]) et aussi parfois des cordons tertiaires [t] qui peuvent s'épaissir et atteindre la taille des cordons secondaires. Les cordons primaires et secondaires sont présents chez tous les exemplaires alors que la présence et le nombre de cordons tertiaires portés par chaque spécimen varient au sein de la population. L'étude de la croissance des cordons spiraux, leur nombre, leur position, leur espacement et leur taille relative sont des critères importants pour la discrimination des espèces. Tous les Campanile examinés montrent comme première séquence sur les tours juvéniles un unique cordon [P2] caréné. Nous avons pu en effet observer sur des coquilles juvéniles de C. giganteum (Lamarck, 1804) de 9 à $11 \mathrm{~mm}$, sur une série de 78 exemplaires de C. cornucopiae (Sowerby J., 1818) de 7 à $12 \mathrm{~mm}$ et sur un exemplaire juvénile de C.pseudoobeliscus (de Grateloup, 1832) de 5,0 mm (Fig. 3),

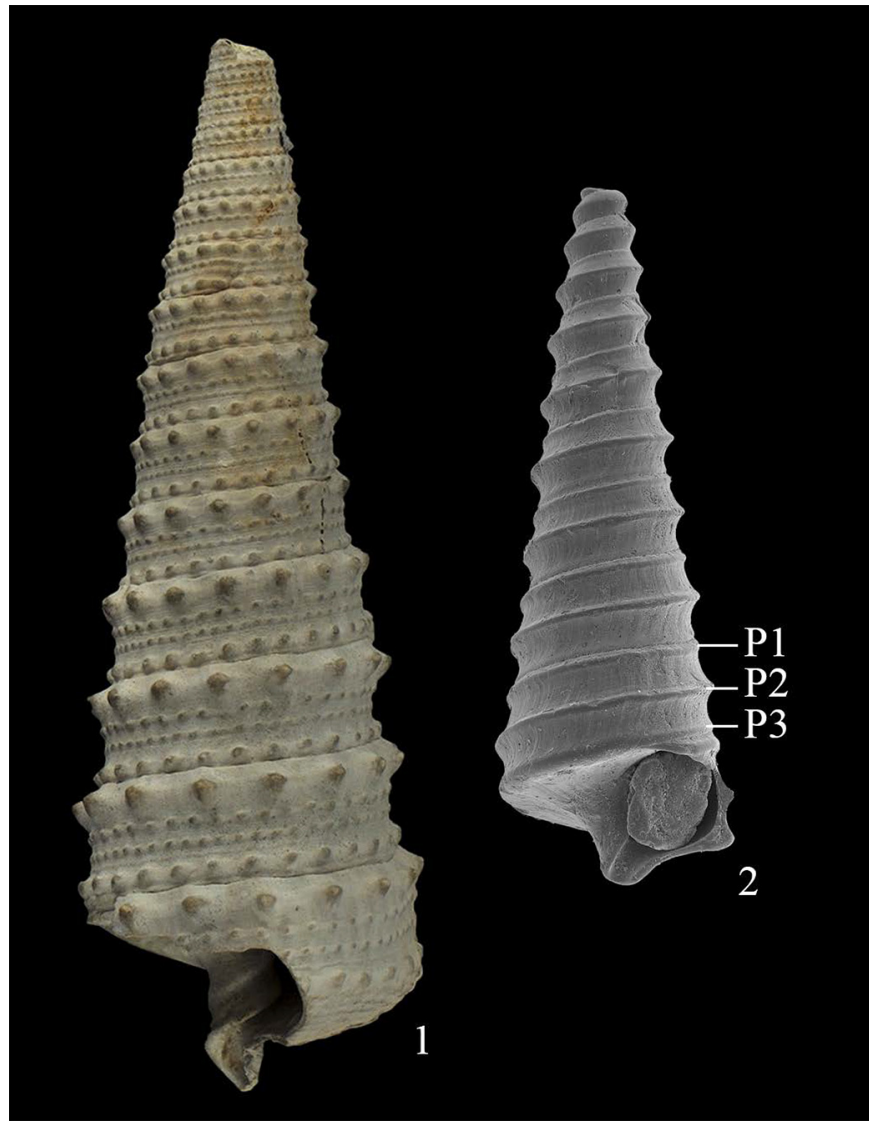

Fig. 3. Campanile pseudoobeliscus (Grateloup, 1832). 1. Aquitanien (Miocène inférieur) de Saucats (Gironde), MNHN.F.A71154 (coll. Vasseur). Hauteur: 92,6 mm. 2. Aquitanien (Miocène inférieur) de Saint-Martin-d'Oney (Landes), spécimen juvénile, MNHN.F.A51211 (coll. Lesport). Hauteur : 5,0 mm.

Fig. 3. Campanile pseudoobeliscus (Grateloup, 1832). 1. Aquitanian (Lower Miocene) of Saucats (Gironde), MNHN.F.A71154 (Vasseur coll.). Height: $92.6 \mathrm{~mm}$. 2. Aquitanian (Lower Miocene) of SaintMartin-d'Oney (Landes), juvénile specimen, MNHN.F.A51211 (Lesport coll.). Height: $5.0 \mathrm{~mm}$.

l'apparition tardive du cordon primaire [P1]; le cordon [P2] seul présent, divisant les tours en deux parties subégales. Avec la croissance de la coquille, le cordon [P1], plus faible que le cordon [P2], devient plus important pour devenir le cordon primaire le plus large puis le cordon orné des tubercules saillants du dernier tour.

\section{Séquence d'apparition et positions des cordons spiraux}

\subsection{Apparition de la première séquence (cordons primaires)}

La comparaison de divers stades de croissance chez plusieurs espèces de Campanile montre que le début de la première séquence d'apparition des cordons spiraux (cordons primaires) ne varie pas d'une espèce à l'autre. Le nombre de cordons primaires portés par chaque spécimen ne varie pas non plus. Il y a seulement trois cordons primaires sur les tours de spires subadultes de la coquille. La terminologie des cordons 
primaires, en tenant compte de l'ontogenèse et de leur correspondance topologique, est celle-ci :

[P1] : cordon primaire placé sous la suture adapicale;

[P2] : cordon primaire placé dans la partie médiane du tour;

[P3]: cordon primaire placé au-dessus de la suture abapicale.

\subsection{Apparition de la seconde séquence (cordons secondaires)}

Après la mise en place des cordons primaires, de nouveaux cordons spiraux s'intercalent. Ces cordons secondaires sont positionnés à égale distance entre deux cordons primaires. Les cordons, faibles au moment de leur formation, s'épaississent au cours de leur croissance. La terminologie des cordons secondaires, en tenant compte de l'ontogenèse et de leur correspondance topologique, est celle-ci :

[s1] : cordon secondaire placé entre les cordons primaires [P1] et [P2];

[s2] : cordon secondaire placé entre les cordons primaires $[\mathrm{P} 2]$ et $[\mathrm{P} 3]$.

\subsection{Apparition de la troisième séquence (cordons tertiaires)}

Après la mise en place des cordons primaires et secondaires, une nouvelle génération de cordons spiraux s'intercale entre les précédents. Les cordons, faibles au moment de leur formation, s'épaississent au cours de leur croissance et peuvent atteindre la taille des cordons secondaires. La terminologie des cordons tertiaires, en tenant compte de leur correspondance topologique, est celle-ci :

[ta] : cordon tertiaire placé entre le cordon primaire [P1] et le cordon secondaire [s1];

[tb] : cordon tertiaire placé entre le cordon secondaire [s1] et le cordon primaire [P2];

[tc] : cordon tertiaire placé entre le cordon primaire [P2] et le cordon secondaire [s2];

[td] : cordon tertiaire placé entre le cordon secondaire [s2] et le cordon primaire [P3].

La terminologie se complète par l'indication de certaine phase dans l'accroissement de la coquille des Campanile (apparition et fusion), nous indiquons donc aussi par des symboles :

[ ] l'apparition d'un cordon;

$[+]$ les cordons qui ont fusionné.

\subsection{Abréviations}

MNHN.F : Muséum national d'Histoire naturelle, Collection de Paléontologie, Paris, France.

IGUT: Institute of Geoscience, University of Tsukuba, Ibaki, Japon.

\section{Systématique}

Classe Gastropoda Cuvier, 1795

Sous-Classe Caenogastropoda Cox, 1960

Sous-cohorte Campanilimorpha Haszprunar, 1988

\subsection{Ordre non défini}

Super famille Campaniloidea Douvillé, 1904

Famille Campanilidae Douvillé, 1904

Genre Campanile Fischer, 1884

Espèce type : Cerithium leve Quoy et Gaimard, 1834 [non

Perry, 1811] par désignation subséquente (Crosse, 1888)

[=Cerithium symbolicum Iredale, 1917 nomen novum]

Origine: Australie, Récent.

Campanile madagasikara nov. sp.

(Fig. 4)

Material type: Holotype MNHN.F.A52029 (coll. Raymond Decary).

Localité type : Cap Amparafaka (Madagascar), Miocène supérieur (Néogène).

Derivatio nominis : Île de Madagascar en malgache. Nom donné en apposition.

Dimensions : Hauteur : 124,2 mm - Diamètre : 62,5 mm.

\section{Description et séquence d'apparition des cordons spiraux}

Les spécimens juvéniles de cette espèce sont inconnus. La séquence d'apparition des cordons n'a donc pu être analysée qu'à partir d'une unique coquille ayant déjà atteint une hauteur de $124,2 \mathrm{~mm}$ pour un diamètre de $62,5 \mathrm{~mm}$ parfaitement conservée. La coquille est de grande taille et présente une spire constituée de huit tours préservés, plans et fortement ornés de cordons spiraux épais et noduleux. L'angle apical est de $22^{\circ}$. Les tours sont marqués par une légère dépression adapicale, faiblement concave, située entre la suture adapicale et le bord postérieur du cordon primaire [P1]. Les tours sont marqués par de nombreuses et très fines stries d'accroissement d'orientation fortement opisthocline, développant un contour opisthocyrte, dans la région adapicale. Sur le tour le plus jeune, les cordons primaires [P1] [P2] et [P3] sont tous les trois déjà présents. Le cordon [P1], légèrement en retrait de la suture adapicale, est épais, orné de granules plus gros et plus espacés que ceux du cordon [P2]. Le cordon [P3], de même épaisseur que le cordon [P2], est plaqué contre la suture abapicale. Les cordons [P2] et [P3] sont ornés de nombreuses petites perles arrondies, étirées longitudinalement. Sur ce tour le plus jeune, on note la présence du cordon secondaire [s1]. Il est légèrement orné de petites perles arrondies, proche du cordon [P1] et séparé de ce dernier par un fin sillon. Il est de même épaisseur que le cordon [P2] et en est séparé par un sillon à peine plus large que celui qui le sépare du cordon [P1]. Dans la région abapicale, on note également la présence du cordon secondaire [s2]. Il est légèrement orné de petites perles arrondies, proche du cordon [P3] et séparé de ce dernier par un fin sillon. Il est de même épaisseur que le cordon [P2] et en est séparé par un sillon plus large que celui qui le sépare du cordon [P2]. Sur ce tour, le cordon [P3] est nettement plus faible que les cordons $[\mathrm{s} 1]$, [P2] et [s2]. Sur le deuxième tour préservé, les granules ornant le cordon [P1] deviennent plus forts, épineux et plus espacés. Le cordon [P3] s'est épaissit rapidement et est devenu aussi large que le cordon [s2]; les cordons [s1], [P2], [s2] et [P3] sont maintenant de même épaisseur. Cependant, les perles ornant les cordons [s2] et [P3] sont plus grosses que celles ornant les cordons [s1] et [P2]. Sur ce tour, on notera dans les 


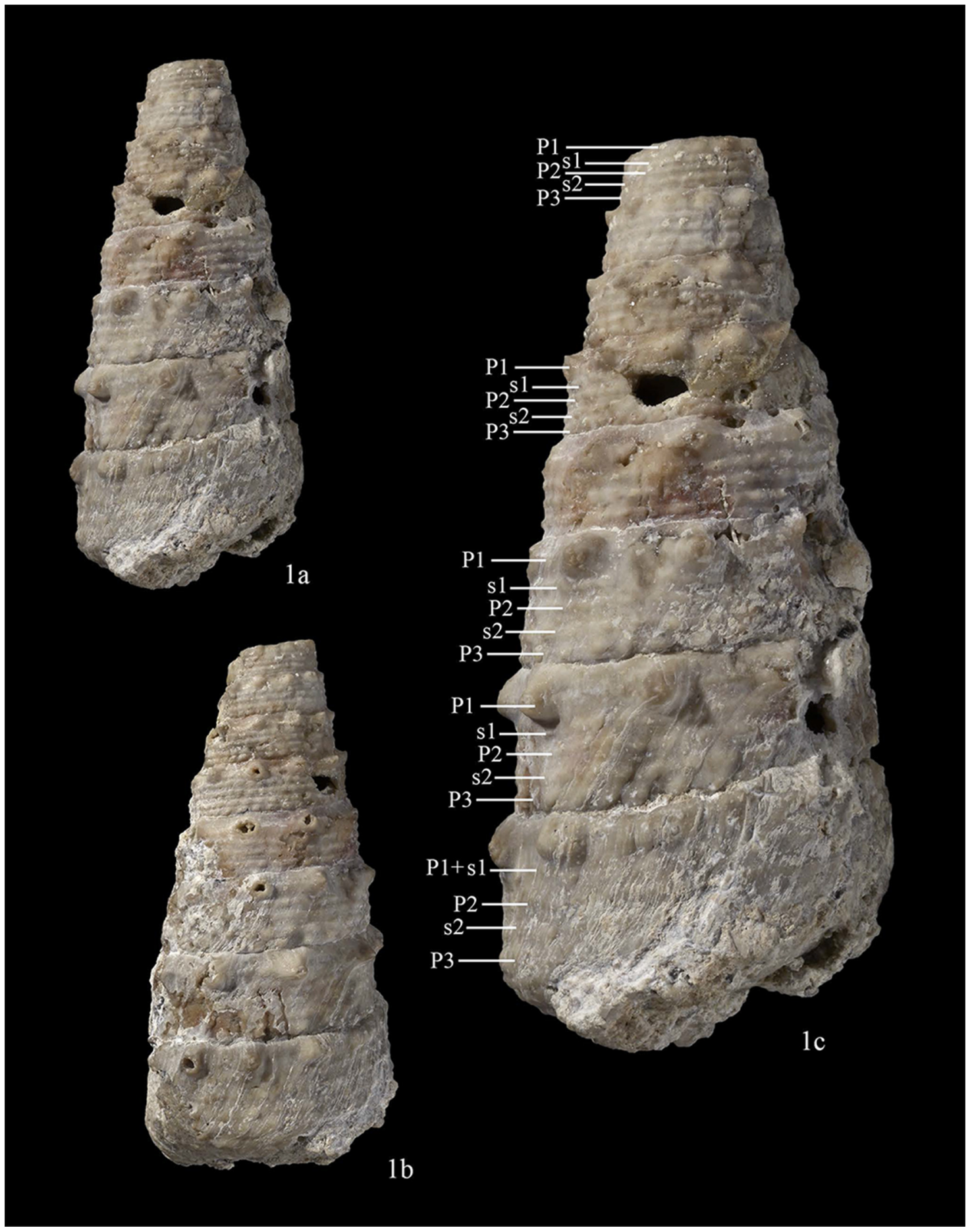

Fig. 4. 1a-c. Campanile madagasikara nov. sp. Miocène terminal du Cap Amparafaka (Madagascar). Holotype MNHN.F.A52029 (coll. Decary). 1c. Identification de la correspondance topologique des cordons spiraux. Hauteur: 124,2 mm.

Fig. 4. Ia-c. Campanile madagasikara nov. sp. Miocene of Cap Amparafaka (Madagascar). Holotype MNHN.F.A52029 (Decary coll.). 1c. Identification of the topological correspondence of the spiral cords. Height: $124.2 \mathrm{~mm}$.

intervalles entre les cordons [s1], [P2] et entre [P2] et [s2] la présence de fins cordons tertiaires. On ne remarque aucun changement dans la séquence d'apparition des cordons spiraux sur le troisième tour préservé en dehors du fait que les granules ornant le cordon [P1] sont encore plus forts, plus épineux et de plus en plus espacés. Sur le quatrième tour préservé, les intervalles entre les cordons [s1] et [P2] et entre [P2] et [s2] sont plus larges, sauf entre les cordons [s2] et [P3] qui sont toujours séparés par un fin sillon. On observe toutefois l'apparition du cordon tertiaire [tc] entre les cordons [P2] et [s2]. On ne remarque aucun changement dans la séquence d'apparition des cordons spiraux sur le cinquième tour 
préservé; le cordon tertiaire [tc] reste aussi faible. Sur le sixième tour préservé les intervalles entre les cordons sont plus importants, même entre les cordons [s2] et [P3]. Le cordon [s1] s'est rapproché du cordon [P1] et les cordons [s2] et [P3] prennent l'ascendant sur les cordons [s1] et [P2] et deviennent plus épais ; les granules ornant ses cordons sont devenus plus gros et plus importants que ceux des cordons [s1] et [P2]. Sur le septième tour préservé, le cordon [s1] se rapproche du cordon [P1] puis se soude à ce dernier. Sur le huitième et dernier tour, au cours de l'accroissement, le cordon [s1] commence à fusionner avec le cordon [P1]. La coquille montre un fragment préservé de la base ornée de cordons concentriques alternant en épaisseur.

\section{Discussion}

Le taxon le plus proche de l'espèce du Miocène supérieur de Madagascar est Campanile hahajimense Noda et Tanaka, 1996 (holotype IGUT 11827) de l'Éocène moyen (Okimura Formation) de l'île Haha-jima au Japon (Noda et Tanaka, 1996 : fig. 3a-d). Cependant, cette espèce présente un cordon primaire [P1] avec une ornementation constituée de granules plus nombreux, moins espacés et de forme quadrangulaire et non d'épines d'orientation abaxiale, espacées les unes des autres. L'espèce malgache est caractérisée par un nombre relativement faible d'épines ornant le cordon primaire [P1], 10 tout au plus, largement espacées, alors que l'espèce japonaise en compte entre 17 et 18 .

Campanile pseudoobeliscus (Grateloup, 1832), espèce de l'Oligocène et du Miocène d'Europe, du Sultanat d'Oman, d'Iran, du Pakistan et de Tanzanie (Fuchs, 1870; Abich, 1882; Cossmann et Peyrot, 1922; Vredenburg, 1828; Harzhauser, 2004, 2007, 2009), est également très proche (Fig. 3). La séquence d'apparition des cordons spiraux est absolument identique à celle de l'espèce C. charpentieri (de Basterot, 1825) de l'Oligocène du bassin d'Aquitaine (Syntype MNHN. F.A71121, coll. Brongniart/de Basterot) dont nous avions donné une analyse complète (Pacaud et al., 2014: 43, fig. 16 fig. 16, pl. 11) et qui est redonnée ici (Fig. 5-6). La coquille juvénile de Campanile pseudoobeliscus (Grateloup, 1832) est ornée seulement d'un cordon primaire [P2], nettement caréné et lisse, partageant les tours en deux parties égales. La partie abapicale des tours est verticale et légèrement concave, la zone adapicale est inclinée à $45^{\circ}$ et relativement plane. Les tours sont marqués par de nombreuses et très fines stries d'accroissement d'orientation orthocline dans la zone adapicale et nettement opisthocline, développant un contour opisthocyrte, dans la région abapicale. Sur une coquille de $5 \mathrm{~mm}$, on observe l'apparition du cordon primaire [P1] vers le quatrième tour de spire, plaqué contre la suture adapicale (Fig. 3) puis l'apparition du cordon primaire [P3], fin, à peine visible, vers le huitième tour de spire, plaqué contre la suture abapicale. Sur des coquilles de C. charpentieri (de Basterot, 1825) ayant déjà atteint une hauteur de $35,0 \mathrm{~mm}$ pour un diamètre de $15,0 \mathrm{~mm}$, les cordons [P1], [P2] et [P3] sont tous les trois déjà présents. Le cordon [P3], peu visible, est souvent masqué par le cordon [P1] du tour suivant. Le cordon [P1], plaqué contre la suture adapicale, est orné de petits tubercules quadrangulaires et le cordon [P2], légèrement plus fin et plaqué contre la suture abapicale, est orné de petites perles arrondies, étirées longitudinalement. La partie médiane entre les cordons primaires est légèrement concave, décussée par les accroissements et ornée de cordons spiraux tertiaires. On note l'apparition du cordon secondaire [s1] à un diamètre d'environ $5,0 \mathrm{~mm}$, il est lisse, fin, proche du cordon [P1]. Il s'épaissit rapidement et s'orne progressivement de petites perles étirées longitudinalement. Le cordon [P1] prend rapidement l'ascendant sur le cordon [P2] est devient plus épais, orné de granules plus grosses et plus espacées que celles du cordon [P2]. Les tours sont très rapidement ornés de nombreux cordons spiraux par démultiplication de micro-cordons autours des cordons [P1], [P2] et [s1]. Sur les tours subadultes d'une coquille de $40,0 \mathrm{~mm}$, à un diamètre d'environ $10,0 \mathrm{~mm}$, on observe l'apparition d'un cordon tertiaire [tb] entre les cordons [s1] et [P2] qui devient très rapidement subégal au cordon [s1]. Il montre une ornementation constituée de petites perles plus fines que celle du cordon [s1] et plus nombreuses. Les cordons [P1], [P2], [s1] et [tb] sont nettement individualisés et nettement espacés entre eux. L'ornementation du cordon [P1] montre des tubercules nombreux, quadrangulaires, alors que les autres cordons restent ornés de nombreux granules plus petits, étirés longitudinalement; le cordon [P3] est simplement décussé par les accroissements. Sur une coquille d'environ $90,0 \mathrm{~mm}$, on compte pour deux tubercules sur [P1], trois granules sur [s1], quatre sur [tb] et deux sur [P2]. La coquille subadulte est turriculée, large en avant, aux tours peu élevés et aux sutures bien marquées.

\section{Implications paléobiogéographiques}

Déjà anciennement, Cossmann $(1906,1908)$ avait résumé la distribution stratigraphique des espèces de Campanile et Wrigley (1940) avait donné quelques occurrences supplémentaires. Plus récemment, Matsubara (2009) a publié un inventaire des espèces cénozoïques. Leur pic de diversité et de répartition a été atteint en Europe au cours de l'Éocène moyen (Cossmann, 1906; Boussac, 1912; Wrigley, 1940; Delpey 1942). Au Paléogène, le genre était répandu dans toute la Téthys avec sa plus grande distribution à l'Éocène. Les espèces sont connues en Europe, en Asie (incluant le Japon), en Afrique et en Amérique du Nord et du Sud, y compris les Caraïbes (Beets, 1941; Jung, 1987 ; Noda et Tanaka, 1996). Après l'Éocène moyen, la diversité des espèces et les dimensions de ce genre ont fortement diminuées et actuellement Campanile est un gastéropode endémique avec une seule espèce connue, C. symbolicum Iredale, 1917, vivant dans des eaux peu profondes en Australie occidentale, de Geraldton à Esperance.

La cause exacte de la quasi-extinction de ce groupe est inconnue. Jung (1987 : 889) considérait qu'il n'y avait pas de trace de Campanile provenant de niveaux d'âge miocène. Néanmoins, les espèces Cerithium charpentieri de Basterot, 1825 et C.pseudoobeliscus Grateloup, 1832, de l'Aquitanien et du Burdigalien, considérées comme des Telescopium depuis Cossmann (1906), sont en fait des Campanile (Pacaud et al., 2014). Après l'Éocène, la distribution du genre s'est rapidement atrophiée au Néogène, de sorte qu'au Miocène, il semble s'être limité à l'Asie du Sud-Est, à la NouvelleZélande, à l'Australie (Ludbrook, 1971; Wakefield, 1977) et à l'Afrique de l'Est, en Tanzanie (Harzhauser, 2009). Ces quatre 


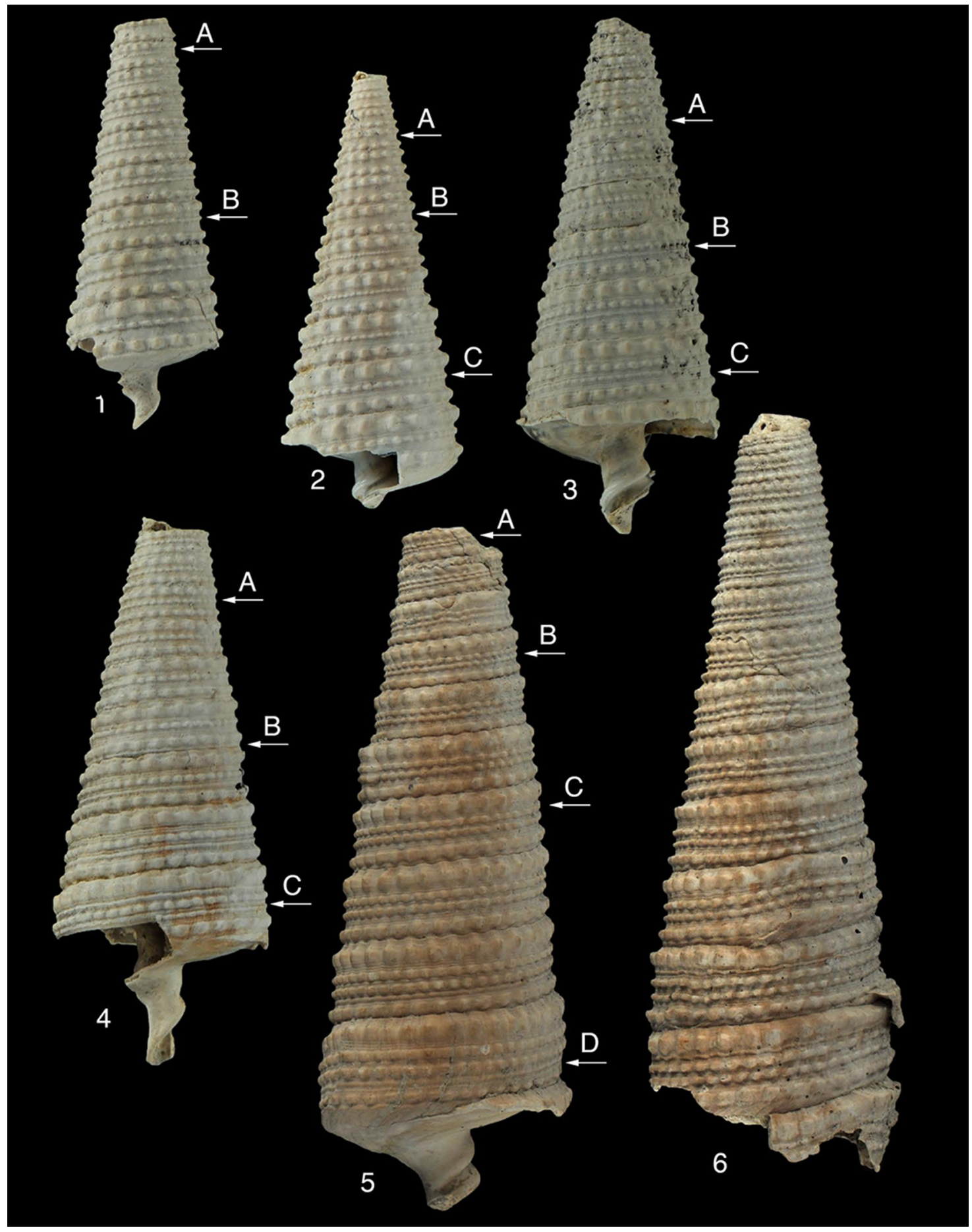

Fig. 5. Campanile charpentieri (de Basterot, 1825). Rupélien (Stampien, Oligocène inférieur) de Gaas (Landes). 1. MNHN.F.A51199 (coll. d'Orbigny). Hauteur : 22,4 mm. 2. MNHN.F.A51200 (coll. d'Orbigny). Hauteur : 26,5 mm. 3. MNHN.F.A51201 (coll. d'Orbigny). Hauteur : 38,7 mm. 4. MNHN.FA51202 (coll. d'Orbigny). Hauteur: 42,5 mm. 5. MNHN.F.A51203 (coll. d'Orbigny). Hauteur: 67,5 mm. 6. MNHN. FA51204 (coll. d'Orbigny). Hauteur : 74,2 mm. L'identification de la correspondance topologique des cordons spiraux des zones A, B, C et D de ces spécimens est donnée dans la figure 6.

Fig. 5. Campanile charpentieri (de Basterot, 1825). Rupelian (Stampian, Lower Oligocène) of Gaas (Landes). 1. MNHN.F.A51199 (d'Orbigny coll.). Height: $22.4 \mathrm{~mm}$. 2. MNHN.F.A51200 (d'Orbigny coll.). Height: $26.5 \mathrm{~mm}$. 3. MNHN.F.A51201 (d'Orbigny coll.). Height: $38.7 \mathrm{~mm}$. 4. MNHN.FA51202 (d'Orbigny coll.). Height: 42.5 mm. 5. MNHN.F.A51203 (d'Orbigny coll.). Height: 67.5 mm. 6.MNHN.F.A51204 (d'Orbigny coll.). Height: $74.2 \mathrm{~mm}$. The identification of the topological correspondence of the spiral cords of zones $A, B$, $C$ and $D$ of these specimens is given in figure 6. 

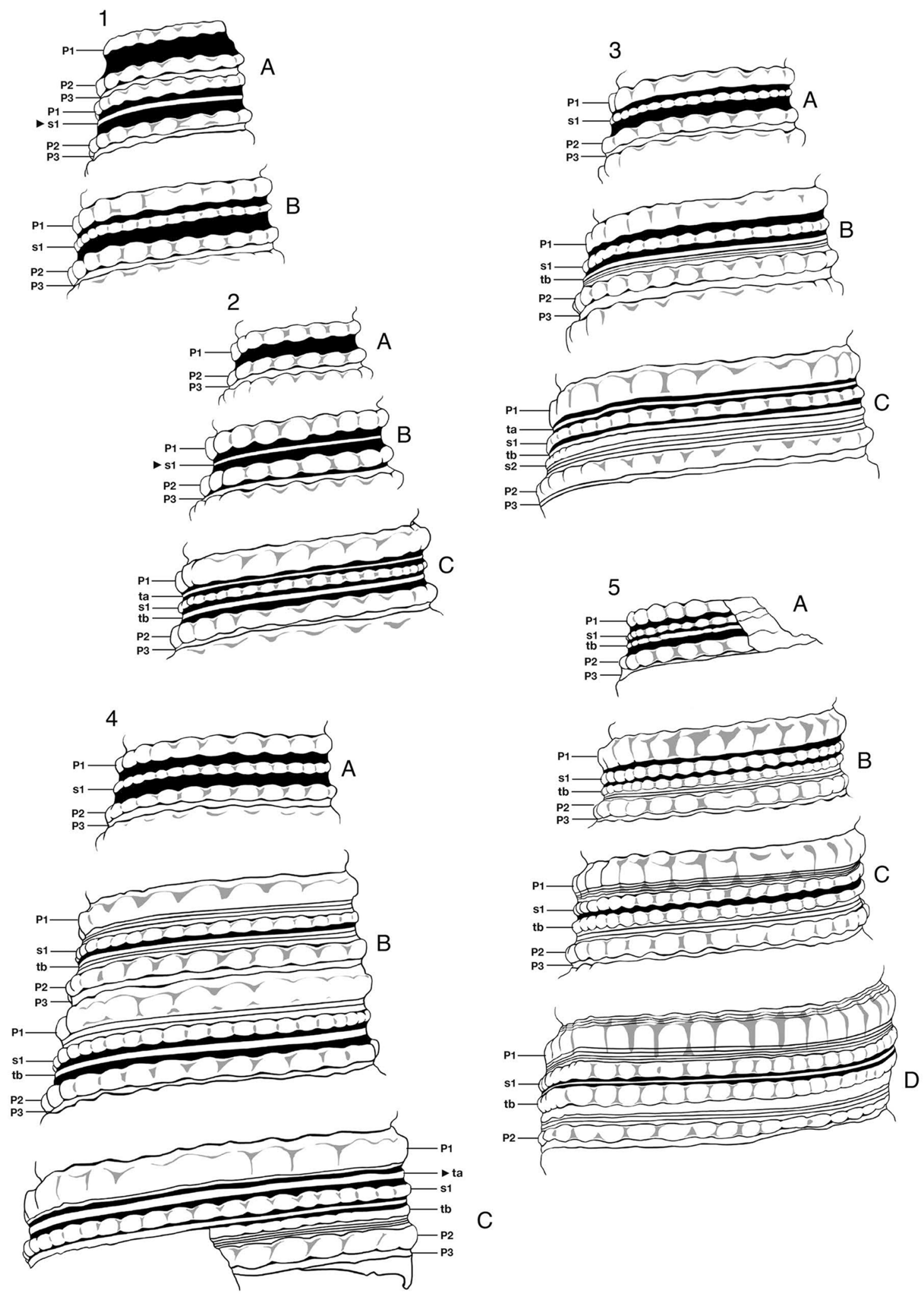

Fig. 6. Nomenclature des cordons spiraux chez Campanile charpentieri (de Basterot, 1825). Les figures A, B, C et D correspondent aux spécimens 1 à 5 de la figure 5 .

Fig. 6. Nomenclature of spiral cords in Campanile charpentieri (de Basterot, 1825). Figures A, B, C and D correspond to specimens 1 to 5 of figure 5 . 
occurrences et la découverte de l'espèce malgache décrite ici suggèrent le maintien de plusieurs aires relictes dans l'océan Indien et le Pacifique au Néogène et jusque dans l'actuel en Australie. L'écologie de l'espèce vivante (Houbrick, 1981) indique que les Campanilidae étaient probablement des herbivores et occupaient la même niche trophique dans les écosystèmes téthysiens d'eau peu profonde que les grands Strombidae actuels (ex.: Strombus, Lentigo, Tricornis, Lambis). Les Strombidae sont apparus à la fin de l'Éocène et au Miocène inférieur et ont prospéré au Pliocène et au Pléistocène inférieur (Abbott, 1960). Nous constatons que l'essor des Strombidae semble concomitant avec le déclin des Campanilidae. La concurrence entre ces deux groupes similaires sur le plan trophique a donc peut-être été un facteur d'extinction des Campanilidae. Au Pliocène, le genre est limité à l'Indonésie (Java) avec C. martini Matsubara, 2009 [=Telescopium gigas Martin, 1881 non Campanile gigas Leymerie, 1851] et à l'Australie avec C.triseriale Basedow, 1902 et C. virginiense Ludbrock, 1971. C. symbolicum Iredale 1917 est également signalée dans les gisements pléistocènes d'Australie (Ludbrook, 1971: 33; Pacaud, 2012: 79, pl. 4, fig. B).

\section{Conclusion}

La morphologie des coquilles du genre Campanile et surtout la méthode descriptive des types de sculpture spirale de plusieurs espèces ont été reconsidérées (Pacaud et al., 2014). Avec l'introduction de cette espèce nouvelle, première occurrence cénozoïque d'un représentant de ce genre à Madagascar, il est de nouveau démontré qu'une méthode descriptive plus rigoureuse basée sur l'identification de la correspondance topologique des éléments sculpturaux de ces espèces et sur l'ontogenèse des cordons spiraux est primordiale et incontournable pour obtenir une meilleure approche de la systématique des espèces de ce genre.

\section{Enregistrement du nouveau taxon :}

Campanile madagasikara: zoobank.org:act:975D3416D03C-4A36-80D5-FB840D0216D9

Remerciements. Nos plus sincères remerciements à Lilian Caze, Philippe Loubry (MNHN/CNRS) et Marie-Béatrice Forel (MNHN) pour les prises de vue et l'infographie des planches, à Bernard Goret pour la préparation du matériel et à Jean-François Lesport pour la coquille juvénile de Campanile pseudoobeliscus qu'il a mise à notre disposition. Nous remercions également Didier Merle (MNHN) et un relecteur anonyme pour leurs critiques sur le manuscrit.

\section{Références}

Abbott RT. 1960. The genus Strombus in the Indo-Pacific. IndoPacific Mollusca 1: 33-46.

Abich H. 1882. Geologische Forschungen in den Kaukasischen Ländern II. Geologie des Armenischen Hochlands, 1. Westhäfte. Hölder, $478 \mathrm{p}$.
Andrianavalona TH, Ramihangihajason TN, Rasoamiaramanana A, Ward D, Samonds KE. 2012. Miocene shark fauna from Nosy Makamby (Mahajanga Basin, Northwestern Madagascar). Journal of Vertebrate Paleontology 32(Suppl. 1): 56.

Baron R, Mouneyres L. 1904. Rapport sur une tournée géologique effectuée en 1903 dans l'Ouest et le Nord-Ouest de Madagascar. Bulletin Économique de Madagascar 4(1): 1-20.

Basse E. 1933. Paléontologie de Madagascar. XVIII : faune malacologique du Crétacé supérieur du Sud-Ouest de Madagascar. Annales de Paléontologie 22: 1-37.

Beets C. 1941. Eine jungmiocäne Mollusken-Faunavon der Halbinsel Mangkalihat, Ost-Borneo (Nebst Bemerkungen über andere Faunen von Ost-Borneo; die Leitfossilien-Frage). Verhandelingen van het geologisch-mijnbouwkundig genootschap voor Nederland en Kolonien, Geologische Serie 13: 1-218.

Besairie H. 1956. Carte géologique [Majunga, $\mathrm{n}^{\circ}$ 2]. Service Géologique de Madagasikara, part : 1 carte : en coul. 59 x $85 \mathrm{~cm}$.

Besairie H, Collignon M. 1971. Géologie de Madagascar. Les terrains sédimentaires. Le Bassin de Mahajanga. Annales Géologique de Madagascar 1: 87-191, pl. 1-14.

Bieler R. 1988. Phylogenetics relationships in the gastropod family Architectonicidae, with notes on the family Mathildidae (Allogastropoda). Malacological Review (Suppl. 4): 205-240.

Bieler R. 1992. Gastropods phylogeny and systematics. Annual Review of Ecology and Systematics 23: 311-338.

Bieler R. 1995. Mathildidae from New Caledonia and the Loyalty Islands (Gastropoda): Heterobranchia. In: Bouchet P, ed. Résultats des campagnes MUSORSTOM, volume 14. Mémoires du Muséum national d'Histoire naturelle 167: 595-641.

Boussac J. 1912. Essai sur l'évolution des cérithidés dans le mésonummulitique du bassin de Paris. Annales Hébert, Annales de Stratigraphie et de Paléontologie du Laboratoire de Géologie de la Faculté des Sciences de l'Université de Paris 6: 1-93, pls. 1-15.

Collignon M, Cottreau J. 1927. Paléontologie de Madagascar. 14. Fossiles du Miocène marin. Annales de Paléontologie 16: 135-170.

Cossmann M. 1906. Essais de Paléoconchologie comparée. $7^{\mathrm{e}}$ livraison. Paris: L'Auteur \& Rudeval, 261 p., 14 pls., 22 text-fig.

Cossmann M. 1908. À propos de Cerithium cornucopiae Sow. Mémoires de la Société Linnéenne de Normandie 23(2), 7: 19-27, pl. 2.

Cossmann M, Peyrot A. 1922. Conchologie néogènique de 1'Aquitaine. Actes de la Société Linnéenne de Bordeaux 73: 5321, pl. 1-7 (espèces n ${ }^{\circ}$ 402-646).

Cottreau J. 1922. Paléontologie de Madagascar. X : fossiles crétacés de la côte orientale. Annales de Paléontologie 11: 111-191.

Delpey G. 1942. Histoire du genre Campanile. Annales de Paléontologie 29: 3-25.

Douvillé H. 1904. Partie 4. Paléontologie. Mollusques fossiles. In: De Morgan J, ed. Mission scientifique en Perse. 3. Études géologiques. Paris: Ernest Leroux, pp. 191-380, pl. 25-50.

Fuchs T. 1870. Beitrag zur Kenntniss der Conchylienfauna des Vicentinischen Tertiärgebirges. Abtheilung I. Die Obere Schichtengruppe, Oder Die Schichten Von Gomberto, Laverda und Sangonini. Denkschriften der kaiserlichen Akademie der Wissenschaften Mathematisch naturwissenschaftliche Classis 30: 137-208.

Harzhauser M. 2004. Oligocene Gastropod Faunas of the Eastern Mediterranean (Mesohellenic Trough/Greece and Esfahan-Sirjan Basin/Central Iran). Courier Forschungsinstitut Senckenberg 248: 93-181, pl. 1-16.

Harzhauser M. 2007. Oligocene and Aquitanian Gastropod Fauna from the Sultanate of Oman and their biogeographic implications for the early western Indo-Pacific. Palaeontographica 280(4-6): 75-121, pl. 1-6. 
Harzhauser M. 2009. Aquitanian gastropods of coastal Tanznia und their biogeographic implications for the early western Indo-Pacific. Palaeontographica 289(4-6): 123-156, pl. 1-5.

Healey JM, Wells FE. 1998. Superfamily Campaniloidea. In Beesley PL, Ross GJB, Wells A, eds. Fauna of Australia, Volume 5. Mollusca: The Southern Synthesis. Melbourne: CSIRO Publications, pp. 733-737.

Houbrick RS. 1981. Anatomy, biology and systematic of Campanile symbolicum with reference to adaptive radiation of the Cerithiacea (Gastropoda: Prosobranchia). Malacologia 21(1-2): 263-289.

Houbrick RS. 1984. The Giant Creeper, Campanile symbolicum Iredale, an Australian Relict Marine Snail. In: Eldredge N, Stanley SM, eds. Living Fossils. New York, Berlin, Heidelberg, Tokyo: Springer Verlag, pp. 232-235.

Hourq V. 1949. Notice explicative sur la Feuille. In: Soalala HI. Carte géologique de reconnaissance à l'échelle du $200000 \dot{e}$, vol. 405. Paris: Imprimerie Nationale, pp. 40-41.

Ikebe N. 1942. Trochid Mollusca Calliostoma of Japan, fossil and recent. Japanese Journal of Geology and Geography 18: 249-282.

Jung P. 1987. Giant gastropods of the genus Campanile from the Caribbean Eocene. Eclogae Geologicae Helvetiae 80: 889-896.

Kiel S, Bandel K, Banjac N, Perrilliat MC. 2000. On Cretaceous Campanilidae (Caenogastropoda, Mollusca). Freiberger Forschungshefte C490: 15-26, pl. 1-2.

Lavocat R, Marie P, Sigal J, 1960. La faune de Foraminifères du Miocène de l'ile Mahakamby (Madagascar). Compte Rendu sommaire des Séances de la Société Géologique de France 11: 211-213.

Ludbrook NH. 1971. Large gastropods of the Families Diastomatidae and Cerithiidae (Mollusca: Gastropoda) in Southern Australia. Transaction of the Royal Society of South Australia 95: 29-42.

Marshall BA. 1995. Calliostomatidae (Gastropoda: Trochoidea) from New Caledonia, the Loyalty Islands, and the northern Lord Howe Rise. In: Bouchet P, ed. Résultats des campagnes MUSORSTOM, volume 14. Mémoires du Muséum national d'Histoire naturelle 167: 381-458, Paris.

Matsubara T. 2009. A Checklist of Species-Group Names of Cenozoic Campanile (Gastropoda: Campanilidae). Paleontological Research 13(3): 285-292.

Merle D. 1999. La radiation des Muricidae (Gastropoda : Neogastropoda) au Paléogène : approche systématique et évolutive. Thèse de doctorat. Paris, France: Muséum national d'Histoire naturelle, pp. 1-499 [inédit].

Merle D. 2001. The spiral cords and the internal denticles of the outer lip in the Muricidae: terminology and methodological comments. Novapex 2(3): 69-91.

Merle D. 2005. The spiral cords of the Muricidae (Mollusca: Gastropoda): importance of ontogenetic and topological correspondences for delineating structural homologies. Lethaia 38: 367-379.

Noda H, Tanaka K. 1996. Tethyan gastropod Campanile fromthe Eocene Okimura Formation in the Haha-Jima Island, Ogasawara Group (Bonin Island), Japan. Professor Hisayoshi Igo Commemorative Volume on Geology and Paleontology of Japan and Southeast Asia. Tokyo: Gakujyutsu Tosho Insatsu, pp. 127-132.

Pacaud J-M. 2012. Un Campanile giganteum (Lamarck, 1804) pêché au large des côtes australiennes; une supercherie du $19^{\mathrm{e}}$ siècle. In:
Les mollusques de l'Éocène du Bassin parisien : un trésor inestimable vieux de 40 millions d'années. Fossiles, Revue française de Paléontologie 3(h.-s.): 72-81, pl. 1-4.

Pacaud J.-M. 2018. Timbellus danapris nov. sp., une espèce nouvelle de Muricidae (Mollusca, Gastropoda) du Priabonien (Éocène supérieur) de Dnipro (Oblast de Dnipropetrovsk, Ukraine). Novapex 19(4): 107-116, fig. 1-4.

Pacaud J-M, Ledon D. 2010. Révision des Mollusques de Blaye (Gironde). 1. Description d'une espèce nouvelle de Turbinidae (Gastropoda, Vetigastropoda). Bulletin de la Société Linnéenne de Bordeaux 145, $\mathrm{n}^{\circ}$ 38(3): 349-359.

Pacaud J-M, Ledon D, Caze, B. 2011. Une nouvelle espèce de Digitolabrum (Gastropoda, Littorinimorpha, Rostellariidae) dans l'Éocène moyen du Bassin d'Aquitaine. Cossmanniana 13: 33-47, pl. 1-4, text-fig. 1-2.

Pacaud J-M, Ledon D, Loubry P, Fernandez S. 2014. Importance de la correspondance ontogénétique et topologique de la sculpture spirale dans la discrimination des espèces du genre Campanile (Mollusca, Gastropoda). Fossiles, Revue française de paléontologie 19: 23-49, fig. 1-17, pl. 1-12.

Perrier de la Bathie H. 1921. Les terrains postérieurs au Crétacé moyen de la région de Majunga. Bulletin de l'Académie Malgache 4: 205-212.

Rakotovao Andrianavah M. 2015. Carte paléontologique de Madagascar. Inventaire et mise en valeur du patrimoine paléontologique. Thèse de Doctorat de l'Université Toulouse 3 Paul Sabatier, Toulouse, 486 p.

Ramihangihajason TN, Andrianavalona TH, Razafimbelo R, Rahantarisoa L, Ali JR, Samonds KE. 2014. Miocene benthic foraminifera from Nosy Makamby and Amparafaka, Mahajanga Basin, northwestern Madagascar. Journal of African Earth Sciences 100: 409-417.

Samonds KE, Zalmout IS, Mitchell MT, Krause DW, Rogers RR, Raharivony LL. 2009. Eotheroides lambondrano, new middle Eocene seacow (Mammalia, Sirenia) from the Mahajanga Basin, northwestern Madagascar. Journal of Vertebrate Paleontology 29 (4): $1233-1243$.

Samonds KE, Fordyce RE. 2019. The first pre-Pleistocene cetacean from Madagascar, western Indian Ocean. Journal of African Earth Sciences 151: 184-188.

Sanders MT, Merle D, Bouchet P, Castelin M, Beu AG, Samadi S, et al. 2017. One for each ocean: revision of the Bursa granularis (Röding, 1798) species complex (Gastropoda: Tonnoidea: Bursidae). Journal of Molluscan Studies 83: 384-398.

Sanders MT, Merle D, Puillandre N. 2019. A review of fossils Bursidae and comments on their use for phylogeny calibration. Geodiverstias 41(5): 247-265.

Vredenburg E. 1828. Description of Mollusca from the Post Eocene Tertiary Formations of North-Western India. Memoirs of the Geological Survey of India 50(5): 1-463.

Wakefield LL. 1977. Lower Miocene Paleogeography and Molluscan Taxonomy of Northland, New Zealand. PhD Thesis. University of Auckland [inédit].

Wrigley A. 1940. The English Eocene Campanile. Proceedings of the Malacological Society of London 24(3): 97-112, text-fig. 1-14.

Citation de l'article : Pacaud J-M. 2020. Étude du genre Campanile Fischer, 1884 (Mollusca : Gastropoda: Campanilidae). 1. Description d'une espèce nouvelle du Miocène supérieur du Cap Amparafaka (Madagascar), BSGF - Earth Sciences Bulletin 191: 21. 